\title{
Substantial pyroelectric effect enhancement in laminated composites
}

\author{
H. H. S. Chang and Z. Huang ${ }^{\text {a) }}$ \\ Department of Materials, School of Applied Sciences, Cranfield University, Bedfordshire MK43 OAL, \\ United Kingdom
}

(Received 7 February 2008; accepted 19 March 2008; published online 16 April 2008)

\begin{abstract}
A mathematical model has been developed to determine the pyroelectric coefficient (PY coef.) enhancement through secondary pyroelectric effect, utilizing a 2-2 connectivity of the pyroelectric lead zirconate titanate (PZT) and elastic laminate layers. Based on the prediction of this analysis, laminar stainless steel (St) PZT/St structures have been fabricated and more than $100 \%$ enhancement in PY coef. has been observed in these structures. Good agreement between the theoretical and experimental results was obtained by taking into account of the stain transfer loss at the St and PZT interface. (C) 2008 American Institute of Physics. [DOI: 10.1063/1.2907701]
\end{abstract}

The pyroelectric (PY) effect concerns the release of the charge upon the change in temperature. It occurs in any material which possesses a polar point symmetry. This effect has been widely used for thermal radiation detection in applications such as fire alarms, intruder detection, and thermal imaging. All PY materials are also piezoelectric, so the pyroelectric coefficient (PY coef.), which is usually measured at constant stress $p^{T, E}$, consists of the primary PY coef. at the constant strain $p^{S, E}$ and the secondary PY coef. arising from strain,

$$
p_{m}^{T, E}=p_{m}^{S, E}+d_{m k l}^{E, \Theta} c_{i j k l}^{E, \Theta} \alpha_{i j}^{T, E}
$$

Here, $d_{m k l}^{E, \Theta}$ is the piezoelectric constant, $c_{i j k l}^{E, \Theta}$ is the elastic stiffness, $\alpha_{i j}^{T, E}$ is the thermal expansion coefficient, $E$ is the electric field, and $\Theta$ is the temperature. Notice the use of Einstein summation convention.

There has been a number of research on the secondary PY coef. over the years. ${ }^{2,3}$ Particularly, the effect of a substrate on PY thin films, arising from thermal expansion mismatch, has been extensively investigated by various researchers. $^{2,4-7}$ Generally speaking, for perovskite-based ferroelectric materials, the product term $d_{m k l}^{E, \Theta} c_{i j k l}^{E, \Theta} c_{i j}^{T, E}$ is much smaller than the primary term $p^{S, E}$, and hence the effect of this mismatch is limited. 4

The possibility of utilizing secondary PY effect to enhance the total PY coef. was suggested by Newnham et al. ${ }^{8}$ and Nan. ${ }^{9}$ Their works led to the development of various composites with superior mechanical flexibility accompanied by good PY performances. ${ }^{10-14}$ They concluded that in most cases due to the small hydrostatic piezoelectric effect, arising from cancellation between coefficients of opposite signs, the enhancement available through the secondary contribution is rather limited. We report in this communication more than $100 \%$ enhancement in PY coef., utilizing a 2-2 connectivity of PY lead zirconate titanate (PZT) and elastic laminate configuration.

A symmetrical configuration, where a PY PZT layer sandwiched between two elastic layers, or vice versa, was adopted to avoid bending (Fig. 1). From thermodynamics, as shown by Nye, ${ }^{1}$ at constant external electric field, the total PY coef. of the system was obtained as (for $i, j, k, l, m=1.3$ )

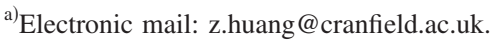

$$
\frac{d D_{m}}{d \Theta}=p_{m}^{T, E}-\left(d_{m k l}^{E, \Theta}\right)\left(c_{i j k l}^{E, \Theta}\right)\left[\alpha_{i j}^{T, E}-\frac{d S_{i j}}{d \Theta}\right],
$$

where $d D_{m} / d \Theta$ is the measured PY coef., $d S_{i j}$ is the strain experienced by PY material, and $d \Theta$ is the temperature change.

It is evident from Eq. (2) that the larger the strain the nonpyroelectric (NP) component can exert on the PY component and the greater the piezoelectric coefficient of the PY material, the bigger the change in the secondary contribution. This leads to the conclusion that stiffer NP material with greater disparity in thermal expansion coefficient $(\alpha)$ with that of PY and more compliant PY material with high piezoelectric coefficients would be desirable. Hence, stainless steel (St) was chosen as the NP material and the PZT for the PY material.

Expression (2) is the general PY coef. for any PY material under strain. With the symmetry of PZT in mind, Eq. (2) can be simplified further to

$$
\begin{aligned}
p_{3}= & p_{3}^{T, E}-d_{31}^{E, \Theta} \sum_{j=1}^{3}\left[\left(c_{1 j}^{E, \Theta}+c_{2 j}^{E, \Theta}\right)\left(\alpha_{j}^{T, E}-\frac{d S_{j}}{d \Theta}\right)\right] \\
& -d_{33}^{E, \Theta} \sum_{j=1}^{3}\left[c_{3 j}^{E, \Theta}\left(\alpha_{j}^{T, E}-\frac{d S_{j}}{d \Theta}\right)\right] .
\end{aligned}
$$

When Eq. (3) is evaluated for a typical PZT, e.g., PZT-5H, ${ }^{15,16}$ the sums of $d_{m k l}^{E, \Theta} c_{i j k l}^{E, \Theta}$ terms for each direction, i.e., corresponding to $d S_{1}$ or $d S_{2}$ and $d S_{3}$, termed as $d c 1$ or $d c 2$, and $d c 3$, are $d c 1=d c 2=-15.9$ and $d c 3=16.0 \mathrm{C} \mathrm{m}^{-2}$. This implies that positive strains in one and two directions,

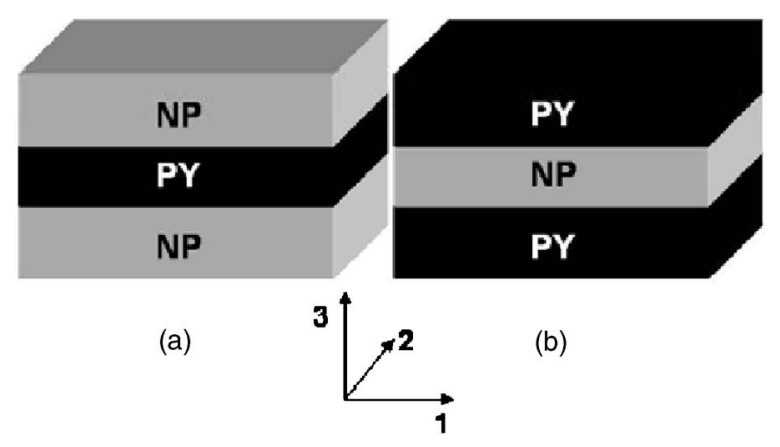

FIG. 1. 2-2 connectivity three layer laminate composite configurations. 
accompanied by a negative strain in three direction, would lead to a larger negative secondary contribution, resulting in the greatest PY coef. enhancement. The best configuration for this requirement is a 2-2 connectivity laminate since, with increasing temperature, it can lead to PZT's strains in one and two axes being positive while strain in three axis becomes negative as a consequence of Poisson effect. Plate theory ${ }^{17}$ and force balance equations were used to elicit the strain that NP can exert on PY. As the system is symmetrical about the 1-2 plane (Fig. 1), two layer plate theory should present a good approximation to our three layer case. From generalized Hooke's law for orthotropic materials ${ }^{17}$ with the assumptions of the Kirchhoff plate conditions, ${ }^{18}$ i.e., only $S_{1}$, $S_{2}, S_{3}$, and $S_{6}$ are nonzero (but $T_{3}=T_{4}=T_{5}=0$ ) and that the shear stress in 1-2 plane, i.e., $T_{6}=\tau_{12}$, is negligible, the total strain experienced by PY can be derived to be

$$
\begin{aligned}
& d^{\mathrm{PY}} S_{1}=d^{\mathrm{PY}} S_{2}=\frac{Y\left(1-2 s_{13} c_{13}\right)\left(1+{ }^{\mathrm{PY}} \alpha d \Theta\right)\left({ }^{\mathrm{NP}} \alpha-{ }^{\mathrm{PY}} \alpha\right) d \Theta}{(1-\nu)\left(c_{11}+c_{12}\right)\left(1+{ }^{\mathrm{NP}} \alpha d \Theta\right) R+Y\left(1-2 s_{13} c_{13}\right)\left(1+{ }^{\mathrm{PY}} \alpha d \Theta\right)}+{ }^{\mathrm{PY}} \alpha d \Theta \\
& \text { and } d^{\mathrm{PY}} S_{3}=\frac{2 Y s_{13}\left(c_{11}+c_{12}\right)\left(1+{ }^{\mathrm{PY}} \alpha d \Theta\right)\left({ }^{\mathrm{NP}} \alpha-{ }^{\mathrm{PY}} \alpha\right) d \Theta}{(1-\nu)\left(c_{11}+c_{12}\right)\left(1+{ }^{\mathrm{NP}} \alpha d \Theta\right) R+Y\left(1-2 s_{13} c_{13}\right)\left(1+{ }^{\mathrm{PY}} \alpha d \Theta\right)}+{ }^{\mathrm{PY}} \alpha d \Theta
\end{aligned}
$$

where $Y$ and $v$ are Young's modulus and Poisson's ratio of $\mathrm{NP}$, respectively, $s_{i j}$ is the elastic compliance of PY, and $R$ is the thickness ratio of PY to NP layers. Evaluating the PY coef. in Eq. (3), with Eq. (4) substituted in, leads to the PY coef. of approximately $-5.0 \times 10^{-4}-\left[\left(6.5 \times 10^{-4}\right) /(0.32 \times R\right.$ +1.0)] $\mathrm{C} \mathrm{m}^{-2} \mathrm{~K}^{-1} \cdot 15,16,19$

The above model assumed that the tertiary PY effect was negligible, the bonding between the layers is perfect, and the thickness of the bonding layers can be neglected. The first assumption is reasonable since our experimental setup is designed to provide uniform heating and cooling. However, due to the finite stiffness and thickness of the epoxy bonding layers and other possible bonding defects, some loss in the transfer of strains between the St and the PZT layer is expected. Therefore, we define an interface coupling factor $k$, similar to that of Bichurin et al., ${ }^{20}$ resulting in the PY coef. of $-5.0 \times 10^{-4}-\left[\left(6.5 \times 10^{-4} \times k\right) /(0.32 \times R\right.$ $+1.0)] \mathrm{C} \mathrm{m}^{-2} \mathrm{~K}^{-1}$, with $k=1$ represent perfect bonding and $k=0$ no bonding at all. Setting $R$ to approach infinity, which is equivalent to no St layer being present, the PY coef. converges to $-5.0 \times 10^{-4}$, namely, $p_{m}^{T, E}$ of a bare PZT-5H. With $R$ approaching zero, which is the case for infinitely thick $\mathrm{St}$ layer or thin PZT layer, the PY coef. converges to its maximum value as the PY coef. of a typical PZT is not dependent on its thickness, while smaller $R$ leads to larger extra strain caused by $\mathrm{St}$ in the PZT layer.

The PY coef. was measured three times for each PZT sample. The first measurement on the bare PZT was followed by yet to be bonded St/PZT/St stack and the final measure-

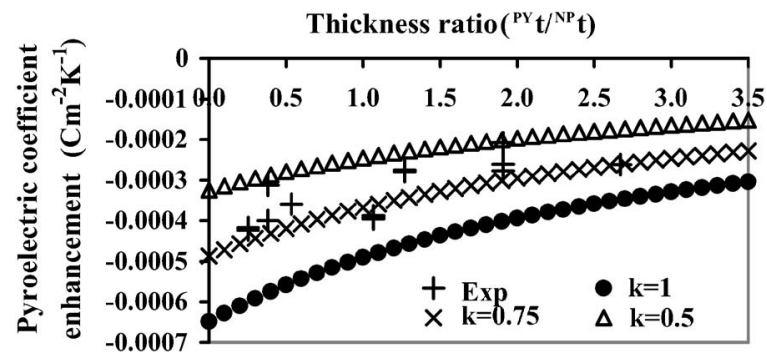

FIG. 2. Magnitude of PY coef. enhancement for the experimental (exp) and theoretical results of PZT-5H. theoretical results of PZT-5H.
Downloaded 05 May 2010 to 138.250 .112 .33 . Redistribution subject to AIP license or PZT-5H in percent.

FIG. 3. Relative PY coef. enhancement for the experimental (exp) and the-

subject to AIP license or copyright; see http://apl.aip.org/apl/copyright.jsp

ment on the epoxy bonded St/PZT/St lamina of that exact stack. This ensured that the only parameter being altered between the observations before and after bonding was the introduction of epoxy bonding. The bare PZT's PY coef. was found to be the same as nonbonded St/PZT/St stack, and the bonded samples were fabricated by applying a well-known commercial epoxy (EPOTEK 301-2, Epoxy Technology, Inc.) onto the surface of each St laminate and placing the PZT between the two, after which the sample was cured at $100{ }^{\circ} \mathrm{C}$ in an oven.

The measured PY coef. enhancement and their theoretical values for the bonded $\mathrm{St} / \mathrm{PZT} / \mathrm{St}$ lamina at different PZT/St thickness ratios $R$ are illustrated in Figs. 2 and 3. For the sample with $R=2.67(267 \mu \mathrm{m}$ PZT-5H with $100 \mu \mathrm{m} \mathrm{St})$, the enhanced PY coef. was observed to be $-6.7 \times 10^{-4}$ compared to $-4.1 \times 10^{-4} \mathrm{C} \mathrm{m}^{-2} \mathrm{~K}^{-1}$ before the bonding. When $R=0.254(127 \mu \mathrm{m}$ PZT $-5 H$ with $500 \mu \mathrm{m} \mathrm{St})$, this rose to $-9.0 \times 10^{-4}$ from $-4.8 \times 10^{-4} \mathrm{C} \mathrm{m}^{-2} \mathrm{~K}^{-1}$ before the bonding. This represents a PY coef. enhancement of 4.2 $\times 10^{-4} \mathrm{C} \mathrm{m}^{-2} \mathrm{~K}^{-1}$ (around 90\%). It is evident from Figs. 2 and 3 that there is a good agreement between the theoretical and experimental values when $k=0.75$, which suggests that the average loss of strain due to interfacial coupling is around $25 \%$. This also implies that there is still a substantial improvement that can be made for our already large enhancement by further perfecting the interfacial bonding. When the PZTs were exchanged to PZT-5A, for the same $R=0.254$, the bonded stack exhibited PY coef. enhancement

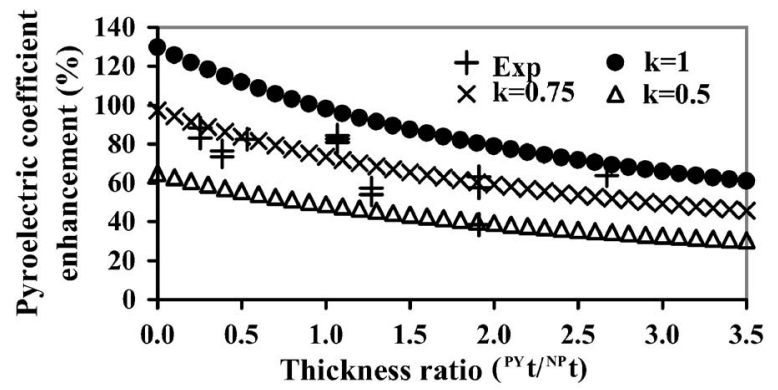


of $105 \%\left(3.9 \times 10^{-4} \mathrm{C} \mathrm{m}^{-2} \mathrm{~K}^{-1}\right.$ gain $)$, an increase from $-3.7 \times 10^{-4}$ to $-7.6 \times 10^{-4} \mathrm{C} \mathrm{m}^{-2} \mathrm{~K}^{-1}$.

In summary, we have observed more than $100 \%$ enhancement in PY coef. in the laminated St/PZT/St structures and interpreted this phenomenon from secondary PY effect. A mathematical model, taking into account the strain transfer loss at the PZT/St interfaces, has also been developed, demonstrating good agreement with the experimental data. This significantly enhanced PY effect could find applications in numerous areas, for example, in PY electricity production ${ }^{21}$ and PY sensors.

We thank Professor R. W. Whatmore of Tyndall National Institute, Ireland and Dr. C. Shaw of Cranfield University for their invaluable discussions on this work. Z.H. thanks EPSRC (EP/D506638/1) for partial financial support.

${ }^{1}$ J. F. Nye, Physical Properties of Crystals (Oxford University Press, Oxford, 1979).

${ }^{2}$ A. Barzegar, D. Damjanovic, N. Ledermann, and P. Muralt, J. Appl. Phys. 93, 4756 (2003).

${ }^{3}$ R. W. Whatmore, Rep. Prog. Phys. 49, 1335 (1986).

${ }^{4}$ J. D. Zook and S. T. Liu, J. Appl. Phys. 49, 4604 (1978).

${ }^{5}$ P. Muralt, Rep. Prog. Phys. 64, 1339 (2001).

${ }^{6}$ Z.-G. Ban, S. P. Alapy, A. Sharma, and J. V. Mantese, J. Appl. Phys. 95, 3618 (2004).

${ }^{7}$ Z.-G. Ban, S. P. Alpay, A. Sharma, and J. V. Mantese, Appl. Phys. Lett. 84, 4959 (2004).
${ }^{8}$ R. E. Newnham, D. P. Skinner, and E. Cross, Mater. Res. Bull. 13, 525 (1978).

${ }^{9}$ C.-W. Nan, J. Mater. Sci. Lett. 13, 1392 (1994).

${ }^{10}$ H. Taunaumang, I. L. Guy, and H. L. W. Chan, J. Appl. Phys. 76, 484 (1994).

${ }^{11}$ Y. W. Wong, L. S. Tai, Y. M. Poon, K. S. Lam, and F. G. Shin, J. Appl. Phys. 96, 3896 (2004).

${ }^{12}$ D. Zhang, B. Yu, K. Zheng, F. Yang, and Z. Li, J. Appl. Phys. 94, 2553 (2003).

${ }^{13}$ F. G. Shin, B. Ploss, H. L. W. Chan, K.-H. Chew, and C. L. Choy, J. Appl. Phys. 94, 1134 (2003).

${ }^{14}$ Y. Yang, L. W. Chan, and C. L. Choy, J. Mater. Sci. 41, 251 (2006).

${ }^{15}$ For the detailed mechanical properties of both PZTs, data from Ferroperm piezoceramics for the corresponding Ferroperm PZTs, namely, Pz27 for PZT-5A and Pz29 for PZT-5H, were used (http://app04.swwwing.net/ files/files/ferroperm\%20matdata.xls, 6 February 2008).

${ }^{16}$ For the thermal and piezoelectric properties of PZT-5H (PSI-5H4E) and PZT-5A (PSI-5A4E), values provided by Piezo Systems Inc. were used. (http://www.piezo.com/prodmaterialprop.html, 6 February 2008).

${ }^{17}$ H. Sol, G. Roebben, W. Heylen, T. Lauwagie, Y. Shi, and O. Van der Biest, NDT \& E Int. 36, 487 (2003).

${ }^{18}$ A. E. H. Love, Mathematical Theory of Elasticity, 4th ed. (Cambridge University Press, Cambridge, 1927).

${ }^{19}$ For the material properties of stainless steel, namely, stainless steel-15$7 \mathrm{PH}$, from GoodFellow (http://www.goodfellow.com/csp/active/ gfhome.csp, 5 February 2008).

${ }^{20}$ M. I. Bichurin, V. M. Petrov, and G. Srinivasan, J. Appl. Phys. 92, 7681 (2002).

${ }^{21}$ R. B. Olsen, D. A. Bruno, and J. M. Briscoe, J. Appl. Phys. 58, 4709 (1985). 